\title{
DETC2003/DTM-48671
}

\section{INTELLIGENT AGENTS IN DESIGN}

\author{
Zbigniew Skolicki \\ George Mason University \\ School of Information Technology \& Engineering \\ 4400 University Drive \\ Fairfax, VA 22030 \\ USA \\ 703-993-3919, 703-993-1710, zskolick@gmu.edu
}

\author{
Tomasz Arciszewski \\ George Mason University \\ School of Information Technology \& Engineering \\ 4400 University Drive \\ Fairfax, VA 22030 \\ USA \\ 703-993-1513, 703-993-1521, tarcisze@gmu.edu
}

\begin{abstract}
In this paper we argue that learning or adaptation ability should be included in the basic set of features characterizing an intelligent agent in design. We propose a collection of attributes describing agents, which are grouped into several categories. Next, we present the results of a detailed study of all agents for design, which were discussed during the First International Workshop on Agents in Design at MIT in August of 2002. A statistical analysis of their attributes has been conducted and its results are reported to suggest future evolution of agents in design. Finally, we briefly overview the topic of Directed Evolution and use its paradigms to predict further development of agents in design. The paper also provides our initial conclusions and suggests further research.
\end{abstract}

\section{INTRODUCTION}

The word agent is commonly understood to be an entity acting on behalf of another entity. Similarly, a software agent is usually described as a computer system to which a user can delegate tasks. Such computer systems are expected to be operational over relatively long time periods and to be at least semi-autonomous. The difference between an agent and a program is not only technical, but also social. An agent is a program that is perceived more as an organism, or autonomous entity, whereas a program is just a tool. Thus, most of the agent's features can be implied from this statement. An agent, being a self-contained unit, must not entirely rely on the external control, but rather take initiative on its own. It has to actively look for information (for example by querying, asking, using sensors) and take actions to affect the environment (or other agents). Minsky claims that intelligence emerges from such interaction and that intelligence is observable only "in the eye of the beholder [1].

While a program can be described in simple terms as having a sequence of instructions, an agent would rather have a constant loop of "intelligent" interactions with the environment. Not surprising, many programs that are not called "agents" by their authors, like some UNIX demons or computer viruses, might be reckoned into this category [2].

The history of intelligent agents started with creating the term "agent" by Oliver G. Selfridge at MIT in the end of the 1960s. Since then the idea has expanded and grown into a diversified discipline. The range of agents starts from simple service programs offering help with limited tasks that could be conducted on behalf of a user, like gopher [3]. Such programs are completely user-driven and hence they have a minimal level of autonomy (they may only decide on technical details of how to perform the task they were created for). The next category is constituted by programs which also serve as a tool to perform a particular task - however this time the autonomy is stronger and much more distinct, since these agents must take high-level decisions about how to reach the goals - as in the case of a flight-booking agent. Finally, there is a category of agents that act on their own, setting goals by themselves and taking initiative in their interactions with a user. Such agents could learn the preferences of the user and give him/her various suggestions.

\section{INTELLIGENT AGENTS}

There are various definitions of an intelligent agent (IA). Since many researchers have been involved in the work on IAs in different contexts, their definitions usually reflect their particular understanding or application of an agent. The term "intelligent agent" is to some extent synonymous with the term "software agent," because "intelligence" is considered to be an immanent part of every software agent [4]. Over the past several years, there have been many attempts to characterize intelligent agents in a formal way. One of the possibilities is to describe agents in terms of attributes and their values, as done for example in works by Wooldrige and Jennings $[3,5]$. 
The following features characterizing intelligent agents can be identified [6]:

- autonomy, decision making - an agent should be at least partially independent, have some goals (internal state), and be able to make decisions $[7,8]$.

social ability, communication and cooperation - an agent should be able to communicate with other agents, or humans, and interact with them to achieve a goal, possibly in a mixed-initiative way $[7,8]$.

- monitoring, perceiving - an agent should sense and observe the environment within which it is located [7, 9].

- acting, operational control - an agent should perform chosen actions which may change the environment or agent's state $[7,9]$.

- reactivity - an agent should respond to the changes in the environment [8]. This may encapsulate the two previous features.

- knowledge, ontology - an agent needs to have an understanding of the environment to perform the task [10]. It can use some kind of ontology (or a concept map) to store and maintain its knowledge.

- learning - an agent should enhance its behavior during its life $[10,11]$.

- continuity - some researchers require an agent to be able to run a process continuously [2].

- mobility - others require an agent to be able to change it's location [3].

The above listed features can be classified as belonging to one of the 4 categories:

- Sensing. An agent reacts to the signals from outside. This includes such features as social ability, communication, perceiving, monitoring etc.

- Reasoning. An agent "lives" (continuity), and "thinks" (autonomy, decision taking, reactivity).

- Learning. An agent "remembers" (knowledge, ontology) and may adapt (learning).

- Acting. An agent sends messages or acts in its environment. This includes acting, operational control, mobility.

Obtaining stimuli from the outer world is the basis for reactive behavior, while reasoning and internal state of an agent are important for its deliberative behavior. Both reactive, deliberative and hybrid agents are described in the literature. Results of an extensive survey of various agents have been provided by Wooldridge and Jennings [8].

Franklin [2] gives the following definition of an agent:

An autonomous agent is a system situated within and a part of an environment that senses that environment and acts on it, over time, in pursuit of its own agenda and so as to effect what it senses in the future.

Again, autonomy is a common feature for nearly every agent, so the above definition can be simply understood as a definition of an agent. Whereas we agree with the above definition in general, we think that for an intelligent agent the definition should be formulated somewhat differently. An agent is usually understood as a closed and non-transparent entity with its own goals or even beliefs. In many cases, an agent must adapt its behavior to the changing or unknown environment, especially if the lifespan of the agent is relatively long. Thus the notion of learning is, if not crucial, then at least very important. In the next section we will show some statistical data confirming our hypothesis for IA in design. We propose the following definition:

An intelligent agent is an autonomous system situated within an environment, it senses its environment, maintains some knowledge and learns upon obtaining new data and, finally, it acts in pursuit of its own agenda to achieve its goals, possibly influencing the environment.

We have used the word "autonomous" to underline that every intelligent agent must take some (if not all) of the initiative. We don't mention directly influencing the agent senses in the future, since this is included in realizing agent's goals.

\section{AGENT ATTRIBUTES}

We will describe agents using binary attributes. As proposed in [5], we will first separately specify attributes for agents and for agent systems (which are understood as systems naturally implementable using agent paradigm). Such systems have been analyzed in the literature [12]. Jennings et al. in their general paper on agents [13] present the history of the development of agents which is a helpful reference for analyzing agents' attributes.

A study of the literature mentioned so far allowed us to propose here a relatively large set of 27 attributes describing agents. At this stage of understanding of intelligent agents for design, we believe that it is desirable to use a wide spectrum of descriptors in order to obtain the maximum discernability of the proposed attributes. The attributes are presented in Table 1 and similar ones are clustered using our background knowledge. We proposed names for the individual groups. The used knowledge elicitation technique has resulted in the distinguishable main characteristics of an agent. The explanation of the attributes is given below.

1. Information. This attribute specifies whether agents store information locally, or have access to some common, shared memory.

2. Perspective. This attribute specifies whether agents can only observe a part of the environment (usually close to them) and/or communicate only with some other agents, or can probe the whole environment and all other agents.

3. Resources. This attribute denotes whether every agent has its own resources and is independent from the others or the agents share some resource (like space, for example).

4. Goal. Agents can have individual goals or they can have one, common goal.

5. Autonomy. This attribute specifies whether a given agent acts on its own, without external stimuli, or it interact with humans. 


\begin{tabular}{|c|c|c|c|}
\hline $\begin{array}{l}\text { Attribute } \\
\text { no. }\end{array}$ & Attribute name & 0 & 1 \\
\hline \multicolumn{4}{|c|}{ Interaction Range (Sensing, Acting) } \\
\hline 1 & Information & Individual & $\begin{array}{l}\text { Shared } \\
\text { (Distributed) }\end{array}$ \\
\hline 2 & Perspective & Local & Global \\
\hline 3 & Resources & Individual & Shared \\
\hline 4 & Goal & Individual & Common \\
\hline 5 & Autonomy & $\begin{array}{l}\text { Acting on its } \\
\text { own }\end{array}$ & $\begin{array}{l}\text { Interacting } \\
\text { with user }\end{array}$ \\
\hline 6 & Competitiveness & Cooperating & Competing \\
\hline 7 & Mobility & Steady & Mobile \\
\hline \multicolumn{4}{|c|}{ Interaction Depth (Reasoning) } \\
\hline 8 & Roles & $\begin{array}{l}\text { Assigned, } \\
\text { different }\end{array}$ & $\begin{array}{ll}\text { No } & \text { roles } \\
\text { assigned } & \\
\end{array}$ \\
\hline 9 & Reaction & Reactive & Deliberative \\
\hline 10 & Speed & Real time & Simulated time \\
\hline 11 & Empathy & $\begin{array}{l}\text { Not modeling } \\
\text { other agents }\end{array}$ & $\begin{array}{l}\text { Modeling other } \\
\text { agents }\end{array}$ \\
\hline 12 & Transparency & $\begin{array}{l}\text { State } \\
\text { communication }\end{array}$ & $\begin{array}{l}\text { Internal state } \\
\text { communication }\end{array}$ \\
\hline 13 & Commitment & $\begin{array}{l}\text { Most } \\
\text { commitment } \\
\text { design }\end{array}$ & $\begin{array}{l}\text { Least } \\
\text { commitment } \\
\text { design }\end{array}$ \\
\hline \multicolumn{4}{|c|}{ Learning (\& Knowledge) } \\
\hline 14 & \begin{tabular}{|l|} 
Language \\
\end{tabular} & Fixed & Learned \\
\hline 15 & Trust level & $\begin{array}{l}\text { Assumes that } \\
\text { all information } \\
\text { is true }\end{array}$ & $\begin{array}{l}\text { Accepts } \\
\text { possible } \\
\text { contradictions }\end{array}$ \\
\hline 16 & Ontology usage & $\begin{array}{l}\text { No, simple } \\
\text { data structures }\end{array}$ & Yes, ontologies \\
\hline 17 & Adaptivity & Non-learning & Learning \\
\hline 18 & \begin{tabular}{|l|l|} 
Initial & \\
Familiarity \\
(with other \\
agents )
\end{tabular} & Yes & $\begin{array}{l}\text { No, to be } \\
\text { learned }\end{array}$ \\
\hline \multicolumn{4}{|c|}{ Structure } \\
\hline 19 & \begin{tabular}{|l|} 
Stability \\
\end{tabular} & Stable & Evolving \\
\hline 20 & Layers & $\begin{array}{l}\text { One-layer } \\
\text { (flat) }\end{array}$ & $\begin{array}{l}\text { Multi-layer } \\
\text { (hierarchical } \\
\text { structure) }\end{array}$ \\
\hline 21 & Diversity & Homogeneous & Heterogeneous \\
\hline 22 & Reusability & One-purpose & $\begin{array}{l}\text { Reusable } \\
\text { modules }\end{array}$ \\
\hline 23 & \begin{tabular}{|l} 
System \\
Flexibility
\end{tabular} & Closed system & Open system \\
\hline 24 & \begin{tabular}{|l|} 
Agent \\
Flexibility \\
\end{tabular} & Closed agent & Open agent \\
\hline \multicolumn{4}{|c|}{ Quantity } \\
\hline 25 & Amount & Many & 1 \\
\hline 26 & Swarm? & Many & Few \\
\hline 27 & Emergence & Yes? & No \\
\hline
\end{tabular}

Table 1. Agents' attributes
6. Competitiveness. This attribute specifies whether agents cooperate or compete with other agents to reach the goal.

7. Mobility. In some environments, agents can move or change their perspective through communication with other agents. This attribute takes a "mobile" value in this case. Otherwise it's "steady."

8. Roles. This attribute denotes whether agents have some specific tasks assigned a priori, or assigned while the system is running

9. Reaction. This attribute describes whether agents react immediately and in a simple way (they are reactive), or they perform more sophisticated actions (deliberative).

10. Speed. If the agent must respond in a specified time to the external, real world input it is operating in real time. Otherwise we assign simulated time value.

11. Empathy. This attribute denotes whether the agents are complex enough to try to model other agents (and predict their behavior).

12. Transparency. This attribute determines whether the internal state of the agent, possibly with its line of reasoning, is visible outside, or whether agent communicates only predetermined states.

13. Commitment. Some agents, or systems, postpone obtaining detailed solution, or keep several solutions from which to choose later. In such case the attribute takes the least commitment value. Otherwise most commitment is assigned.

14. Language. This attribute specifies whether the agent learns or modifies the language while running.

15. Trust level. This attribute specifies whether contradictions are possible in the system and whether the system has some way to deal with it.

16. Ontology usage. This attribute denotes whether agents use some kind of ontology to represent concepts from the domain. If agents use some complex database but it does not resemble an ontology, the "simple" value is assigned.

17. Adaptivity. This attribute describes whether agents or the system adapt or learn something.

18. Initial familiarity (with other agents). Sometimes the relations and communication paths between agents are set before running and are stable. In this case we assume agents "know" each other initially. Alternatively agents can dynamically discover and interact with others, as in the case of hundreds of anonymous agents or dynamical environments.

19. Stability. The attribute specifies whether agents have stable architecture, or whether they can reconfigure in the runtime. 
20. Layers. Sometimes agents are hierarchically organized and thus constitute a higher order system, often with top agents delegating the tasks to the underlying ones. Also, in some cases agents can communicate through higher-level agents serving as managers. Such cases determine the hierarchical structure. In other cases the agents are all equal, negotiating with each other on a peer level, making a one-layer system.

21. Diversity. If all agents are the same (at least initially) they are homogeneous, otherwise they are heterogeneous.

22. Reusability. If parts of agents are easily reusable for other purposes (being portable modules), or even whole agents can easily serve different tasks, we assign reusable value to the attribute. Otherwise we have a one-purpose system.

23. System Flexibility. If it is easy to add agents or components to the system, we call such a system "open." Otherwise, it is "closed."

24. Agent Flexibility. If the agent architecture is easily expandable (a characteristic that does not have to actually take place in runtime; that is, a characteristic that would be described by the "stability" attribute) and the components can be easily added or dropped, the agent is "open." Otherwise it is "closed."

25. Amount. The attribute specifies whether there is one or more agents in the system.

26. Swarm? This attribute determines whether there is a large number of agents that they may be considered as a swarm or whether there is only a limited number of relatively easily distinguishable agents.

27. Emergence. It is believed that the huge number of relatively simple operations sewn together may result in a higher level, macroscopic behavior. The Emergence attribute attempts to capture this feature.

As can be seen, the first two groups can be assigned to canonically accepted features of an agent, namely sensing, reasoning and acting. The attributes from the third set, although they could be included into "reasoning" group, seem to better constitute their own group, which we called "learning". Finally we have several attributes dealing with different possibilities of constructing the agent, and we organized them into a "structure" group.

\section{INTELLIGENT AGENTS IN DESIGN}

Intelligent agent for design is simply understood by us as an intelligent agent developed purposely for use in engineering designing process within the conceptual development stage, within the design development stage, or during the both stages.

The history of intelligent agents in design can't be separated from the evolution of knowledge-based computer design support tools. For this reason, several examples of such evolution are provided below.

In the mid-eighties, the Expert Systems Revolution in engineering resulted in pioneer work at Carnegie Mellon University, initiated by Steven Fenves and Mary Lou Maher, on knowledge-based conceptual design support tools [14]. High Rise, an expert system for the conceptual and preliminary design of tall buildings is the first such tool. It can be considered as a design agent, although it didn't have any automatic knowledge acquisition capability. In this case, human design experts had to determine and subsequently manually encoded all design rules.

The concept of an expert system for conceptual designing has been expanded into a concept of a learning expert system for conceptual designing by the second author, working with a computer scientist, Wojciech Ziarko, on the engineering applications of inductive learning based on the theory of rough sets [15]. In the time period 1985-1989, they developed an Intelligent Agent, called "Brzdyl", for the conceptual designing of steel structural systems of tall buildings. It had a learning component based on the symbolic inductive learning utilizing the theory of rough sets. It operated in two stages: learning and production. In the first stage it acquired and modified design rules from examples while in the second stage the acquired knowledge was used for "filtering" design concepts that were randomly generated. The system, as well as the results of initial design experiments, have been described in several papers $[16]^{1}$.

Over the last year or two, the reemergence of interest in Intelligent Agents in the context of engineering design can be observed. For example, in the Summer of 2002, the First International Workshop on Agents in Design was organized, by John S. Gero and Frances MT Brazier, at MIT [17]. This workshop attracted the majority of researchers investigating agents in design and produced proceedings containing papers describing various agent systems for design. The collection of these papers is an excellent reflection of the state of the art. Therefore, it has been used by the authors to study agents in design and to attempt to predict their expected evolution. Although the randomness of the sample cannot be formally proved, we believe that it is sufficient for the purpose of a preliminary study, because of the variety of agents included.

In total, an extensive study of all 17 agent systems has been conducted [18-34] and for each system a string of numbers representing it in the context of the above attributes has been created. The characteristics of every agent can be presented as a binary vector of length 27 . We assigned the "0" and " 1 " values to the attributes in such a way that " 0 " denotes a simpler behavior and " 1 " denotes a more complex and sophisticated behavior (except the cases of "speed", "amount", "swarm" and "emergence" attributes). In doubtful cases, we assigned value "0.5." We expected that the agents would either have mostly 0's (which would be many simple, interacting agents, possibly making up a swarm and having an emergent behavior) or mostly 1's (which would be a smaller number of intelligent agents where the intelligence comes from elaborated algorithms rather than from the emergence).

To our surprise, though, the analysis has revealed that the distribution of attributes' average value for the considered class of 17 agents is closer to a normal distribution rather than to a

\footnotetext{
${ }^{1}$ Interestingly, the second author submitted two proposals to the National Science Foundation in the late 1980s. Unfortunately, at that time the idea of autonomous design support tools was considered too esoteric and radical. Therefore, his proposals were met with disbelief and twice rejected as premature and too speculative.
} 
bi-modal one. This would suggest that the values for separate attributes may be chosen independently. The histogram is shown in Fig. 1. A Q-Q plot against the normal distribution is shown in Fig. 2. The $\mathrm{R}^{2}$ value for linear regression is equal to 0.97 .

The analysis of particular attributes lets us draw further conclusions. The chart in Fig. 3. shows an average value (with confidence level of $95 \%$ and using Student's t-distribution) for every attribute. Whereas some attributes have an average value close to 0.5 suggesting that both possible options are used in different systems, others tend to have a very low or very high average value indicating that current research prefers one type of value. Let us look at the cases where 0.5 is not within the confidence interval and try to draw conclusions about possible useful directions of research.

\section{Histogram}

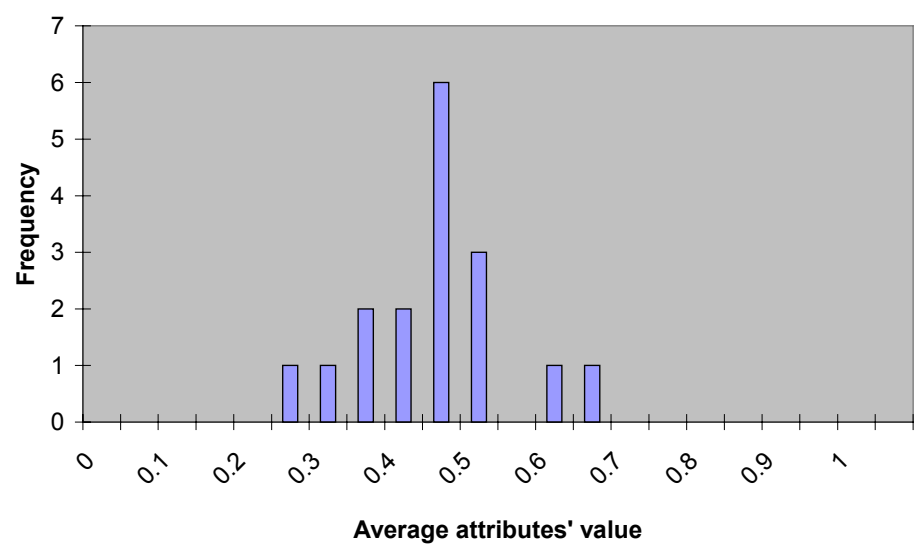

Figure 1. An average value of attributes measured for every case

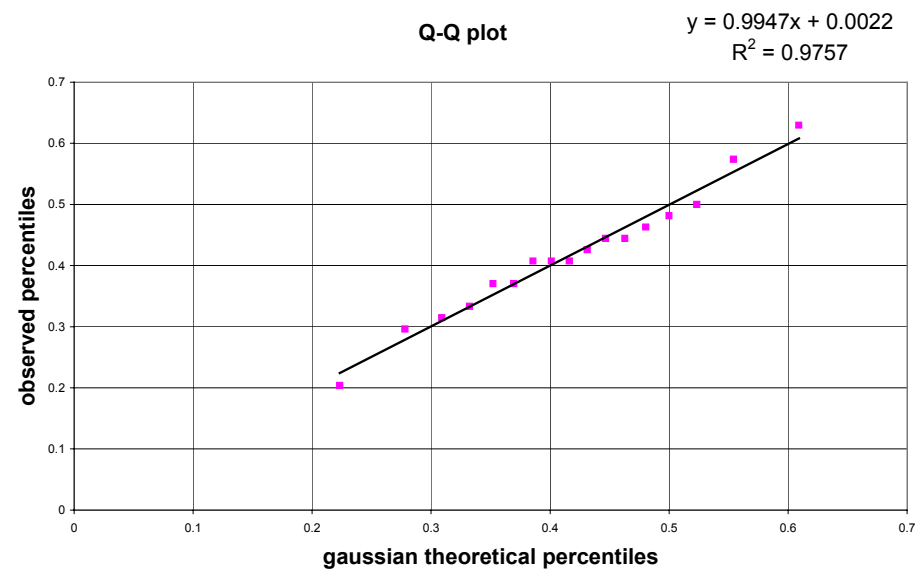

Figure 2. A Q-Q plot against a normal distribution average attribute

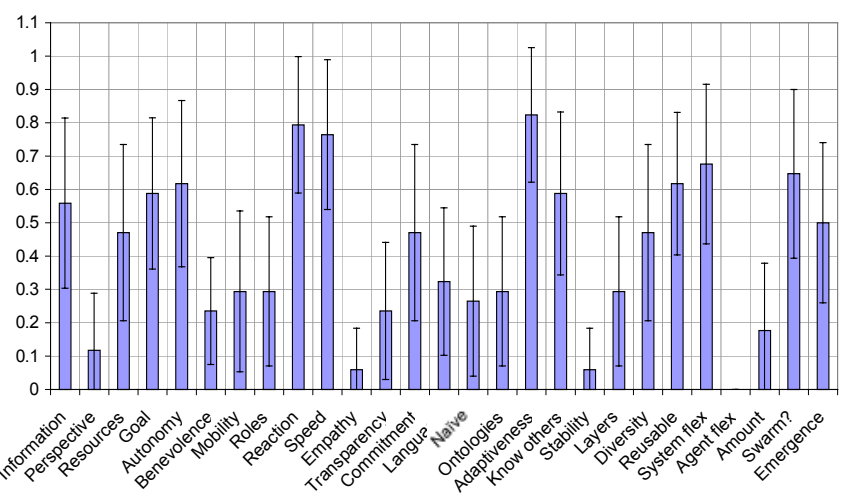

Figure 3. Average attributes' values

a. A low average value of Perspective attribute suggests that in most cases agents are built to act locally with limited ability to probe the environment and with high level of encapsulation. This is not surprising because that is consistent with the nature of agent systems promoting such solutions.

b. A low value for the Competitiveness attribute reveals that the agents mostly cooperate rather than compete. New research in competitive co-evolutionary algorithms could raise the value.

c. A high level for the Reaction attribute shows that the agents are modeled to have a relatively high level of sophistication. Research in swarm environments could significantly change the situation.

d. Probably because of the previous comment, agents usually work in a "simulated time" (Speed attribute), meaning that they do not have to immediately react to the input from the real word. Transferring the systems into robotics would probably change this situation.

e. A very low value of Empathy suggests that although the agents use elaborate techniques, they do not put much effort into modeling other agents, probably because the benefits are smaller than the costs. With growing complexity of systems, however, this feature could be required.

f. Similarly, it seems that the agents don't show their internal state outside (Transparency attribute). This may be the result of encapsulation and the nature of agents systems.

g. Most agents assume the information as true (Trust level attribute). This is not the case, however, in the real world and also in robotics. Hence, research in this direction should be useful.

h. A high level for the Adaptivity attribute shows that most agents or systems acquire knowledge while running. This is what we claim is necessary for an intelligent agent, and therefore this value is not surprising for us (note that this attribute has the highest value) and supports our previous statements.

i. A very low value for Stability and Agent Flexibility shows an interesting direction for research. One can easily imagine agents evolving in runtime as they adapt to the environment (the first attribute), or adding 
and dropping components as needed - either before the system starts or also during runtime.

j. Finally, in most agent systems considered, we have more than one agent (Amount attribute). We believe that it is the right direction and consistent with the philosophy behind agent systems evolution in which the interaction plays a major role. It is worth noting that even in the case of a single agent, the user usually plays the role of a second agent and thus the interaction is also important.

Another interesting analysis comes from comparing the average attributes for agent systems classified as swarms and non-swarm systems. Although in general the average value for all attributes does not differ much (as mentioned before), looking at them one by one provides some interesting observations, mostly confirming our predictions.

In Fig. 4., we provided the chart representing particular attributes for both swarm agents and non-swarm agents (we call them individuals). The following observations can be made about swarm agents as compared to individuals (although not all of them are formally justified statistically - for the figures we have assumed a confidence level of $90 \%$ ).

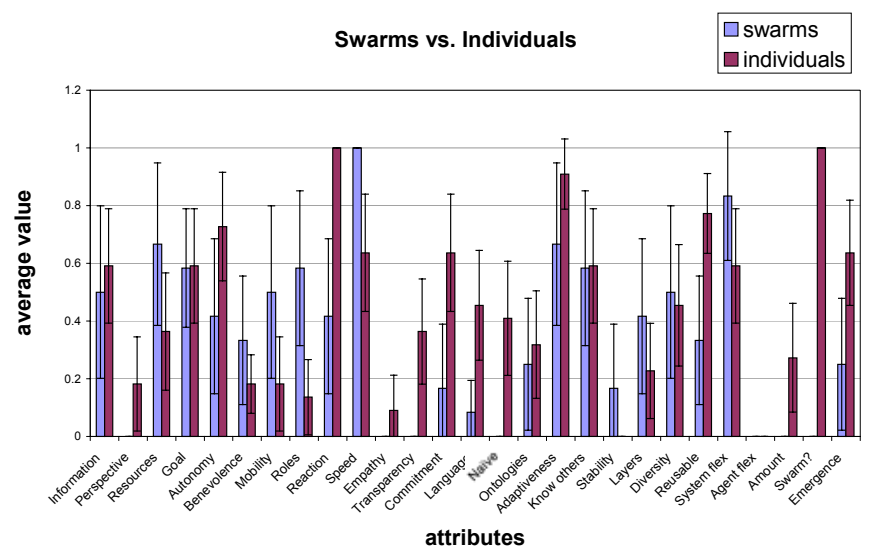

Figure 4. Swarms vs. Individual Agents (Individuals)

a. Swarm agents always act on a more local perspective

b. They tend to share resources to a greater extent

c. They have less autonomy

d. They compete more

e. They are more mobile

f. They tend to discover roles dynamically in runtime

g. They are simpler (more reactive)

h. Surprisingly they tend to act in a "simulated time"

i. They are less transparent

j. They have a fixed language

k. They assume the information to be true

1. They seem to be less reusable

Whereas some of these features seem to be natural because of the way a swarm is built, it seems that the characteristic of swarms learning does not have to be this way. In other words, it would be interesting to build swarm models with a higher level of adaptivity, allowing for more unknown in the world. Also, exchanging information between agents would lead to more cooperative systems.
It is tempting to try to build a system whose genome would be described mostly by 1's (except for the last 3 attributes) that is to make every aspect of the system as sophisticated as possible. However, there is a danger in this undertaking. First, there are still many technical and computational problems to be resolved before it will be feasible. The second, and maybe even more important aspect is that because the interaction of particular subcomponents, it would be extremely difficult, if not simply impossible, to analyze their role. It is probably more reasonable to focus on understanding the behavior and impact of particular elements. Only then the role of interaction and the still unsolved question of emergence could be answered. Such research would hopefully lead to simplifications and rejections of unnecessary, or redundant, components and to the possibility of dynamic management of the needed elements (see the Patterns of Evolution below).

\section{DIRECTED EVOLUTION}

Directed Evolution in its present form has been recently proposed by Dana Clarke [35, 36]. He defines it as a method for "the development of a comprehensive set of scenarios which allow for the planning and ongoing development of future generations of engineering systems for the purpose of financial gain and controlled business growth." It is based on the principle that over time the natural evolution of engineering systems is not random, but occurs according to the objective patterns of evolution. These patterns can be acquired through analysis of patent records and the history of engineering. Subsequently, they can be used in forced, or Directed Evolution for the development of scenarios, each based on a separate line of evolution. Patterns of evolution and the lines of evolution can also be used in studying and evolving intelligent agents in design, and therefore they are briefly discussed in this section.

The concept of Directed Evolution has a long history. It is directly related to the work of Genrich Altshuller [37], who in the 1940's initiated within the Soviet Union research on acquiring design and inventive knowledge from patents. That work led to the development of TRIZ, a Russian acronym for the Theory of Inventive Problem Solving. Even initial versions of TRIZ contained several Patterns of Evolution. However, they were significantly expanded and refined as the result of more recent work at the Kishnev School by Boris Zlotin and Alla Zusman [38], and later continued by them at the Ideation International in this country. Today, there are eight Patterns of Evolution that are also highly relevant to the evolution of intelligent agents in design. The patterns are as follows:

1. Stages of Evolution: all systems evolve according to the S-curve.

2. Evolution toward Increased Ideality:

a. Each system performs functions generating both useful and harmful effects,

b. A system's evolution maximizes ideality, understood as a ratio of useful to harmful effects

c. Inventive designing improves system's ideality.

3. Non-uniform Development of System Elements:

a. Each system component has its own S-curve,

b. Different components usually evolve according to their own schedules

c. Different components reach their inherent limits at various times, resulting in contradictions 
d. The component that reaches its limit first "holds back" the overall system

e. The elimination of contradictions allows the system to improve.

4. Evolution toward Increased Dynamism and Controllability:

Increasing system dynamism and controllability allows functions to be performed with greater flexibility.

5. Increased Complexity then Simplification (Reduction):

Engineering Systems tend to develop first towards increased complexity, and then towards simplification.

6. Evolution with Matching and Mismatching Elements: System elements are matched or mismatched to improve performance or to compensate for undesired effects. A typical sequence: 1. unmatched elements, 2. matched elements, 3. mismatched elements, 4. dynamic matching and mismatching.

7. Evolution toward the Microlevel and Increased Use of Fields: Engineering systems tend to undergo transition from Macrosystems to Microsystems. The transition requires different types of energy fields.

8. Evolution toward Decreased Human Involvement

A line of evolution is a time sequence of previous, present, and future concepts of a given engineering system, for example of a car suspension system. It clearly shows how a given system changes, or evolves, over a long time period, that can extend from months to tens of years. In a line of evolution, the individual design concepts are represented by combinations of symbolic attributes and their values, or by simple sketches identifying their key characteristics. Good examples of various lines of the evolution of complex engineering systems are provided in [37] for airplanes. A more specific and detailed example of a line of evolution, in this case of joints in steel space structures over the period of about 50 years, is provided in [39]. This paper also shows how a line of evolution can be used to acquire design knowledge (in this case for the structural shaping of joints in space structures) in terms of domain specific patterns of evolution.

\section{INTELLIGENT AGENTS EVOLUTION SCENARIOS}

An attempt has been made to consider agents in design in the context of patterns of evolution. These results are briefly described below.

\section{Stages of Evolution}

The ability to acquire knowledge has increased over the last 20 years. Whereas the first systems usually used a predefined knowledge, current systems tend to collect information in the runtime. We have already completed the stage of childhood, which could be represented by early systems like High Rise [14], and we are now in the stage of growth. During this stage many exciting developments and rapid progress are expected. Our observation is consistent with the situation within the Information Technology community, both scientific and applications oriented, where more and more effort is focused on data mining for knowledge and in general on automated knowledge acquisition.

\section{Evolution toward Increased Ideality}

This evolution pattern can also be observed. From the user's (designer's) point of view, an agent in design should have all desirable features. There is no question that subsequently proposed agents for design have a growing number of features. Therefore, the growth of the number of features in the future can be observed.

\section{Non-uniform Development of System Elements}

At this stage of the evolution of agents in design, the occurrence of this pattern cannot be clearly confirmed. Within 3-5 years from now, however, the issue of this pattern should be investigated again. As suggested by previous analysis, agents have a wide variety of attributes. At this stage of their evolution, it is difficult to classify them into unambiguously different classes. This suggests a parallel and independent development of elements taking place in various systems. Although, intuitively we would expect clustering of agents specialized for particular tasks, statistically it is difficult yet to observe, at least in the sub-domain of agents for design.

\section{Evolution toward Increased Dynamism and Controability}

This pattern has been originally proposed in the context of mechanical systems in which initially fixed parts become moveable or adjustable. This pattern also occurs in the case of agents in design. In a number of recently proposed agents, their various components may undergo changes as these systems adapt to their environment. In particular, many agents are mobile, being able to dynamically decide about the next place (environment) to which they will move. Similarly, contrary to a static network of agents, they often dynamically discover one another in systems consisting thousands of them.

\section{Increased Complexity then Simplification}

The first part of this pattern can be easily observed. The agents in design become more complex, and that is typical for the stage of their evolution called "growth." The next 2-3 years will provide an answer if this evolution pattern holds. Based on many discussions with design researchers, however, we can easily predict that very soon the process of simplifications of many agents will start. It will be partially driven by an improved understanding of importance of the individual components of an agent and partially by limitations of the existing programming languages and tools.

\section{Evolution with Matching and Mismatching Element}

The dynamic characteristic of current agent systems makes it possible to properly adjust the level of complexity of particular components in the runtime. Whereas some agents, that reason deliberatively and give more sophisticated answers, may be more proper in specific situations, fast and robust answers are needed in real time systems. It is not difficult to imagine that the systems using managers to coordinate tool agents can monitor their behavior and exchange them dynamically if some feature of a given agent doesn't fall into the acceptable range. 
Also, as we look at the development of agents, we see that although the mechanisms which they use to perform particular tasks may last for longer and be easier for scaling up, the communication, dynamic coordination of agents and learning mechanisms tend to evolve faster. Therefore, although we may still use the same tool agents because the communication subcomponent may not match the others any longer and must be exchanged for a newer one. For example, one may want agents to use KQML or FIPA protocols for communication; standards not before known.

\section{Evolution toward the Microlevel and Increased Use of Fields}

Although the occurrence of this pattern has been difficult to observe thus far, we have already experienced agents working for different "microlevel" tasks like mail filtering. Agents become subordinated to bigger programs that use them only to perform a certain task. That occurrence differs from having agents act as the main system. Design activity becomes an intermediate step to reach some goal, rather than being the result of the system.

\section{Evolution toward Decreased Human Involvement}

The entire history (evolution) of agents in design can be considered as a process driven by, and delivering to, decreased human involvement. Therefore, there is no question that this pattern of evolution has been occurring and should be considered when predicting further progress in the area of agents for design.

At this stage of research, it is difficult to fully support the proposed scenarios or to make a long term prediction along lines of evolution.

\section{CONCLUSIONS}

This paper presents the initial results of a fundamental research project on intelligent agents in design, being conducted in the Information Technology and Engineering School at George Mason University. Our research has clearly revealed, as expected, that the technology of intelligent agents in design is still in early evolution stages. However, our specific results should be useful in building a better understanding of the evolution of intelligent agents in design. Also, they should provide future research directives for design researchers interested in intelligent agents.

In the paper, a class of nominal binary attributes has been proposed to describe agents' systems. Although the description provided may not be complete, it is sufficient to distinguish between various existing (or in development stages) agents for design. The proposed description (intelligent agents for design representation space) has been used to analyze a set of 17 agent systems. The conducted analysis has identified learning ability as one of the most important features of an intelligent agent for design and led us to a new definition of such an agent. By comparing the average values of attributes, we have made a number of predictions about the future directions of research on intelligent agents.

Finally, we have realized that it is premature to use Directed Evolution to predict the evolution of intelligent agents for design, considering the early stages of their evolution and the still limited body of available knowledge. However, we believe that Directed Evolution should soon become a useful tool for making predictions and building lines of evolution, during the next several years when much more is learned about intelligent agents in design.

\section{ACKNOWLEDGMENTS}

The authors gratefully acknowledge support for their research from the NASA Langley Research Center under the grant NAG-1-01030 and from George Mason University that provided the Graduate Interdisciplinary Research Scholarship for the first author during the academic year 2002-2003.

\section{REFERENCES}

[1] Wallace A., Boldyreff C., 1999, “Agents and Agent Based Design Approaches to Engineering Design and Manufacturing", Proceedings of Computer Aided Production Engineering (CAPE'99), Maropoulos, P.G., McGeough, J.A., eds., University of Durham

[2] Franklin, S., Graesser A., 1996. "Is it an Agent, or just a Program? A taxonomy for Autonomous Agents," Proceedings of the Third International Workshop on Agent Theories, Architectures and Languages, Springer-Verlag

[3] Jennings, N. R., Wooldridge, M., 1998, “Applying agent technology", Jennings N. R., Wooldridge, M., eds., Agent Technology: Foundations, Applications, and Markets, Springer-Verlag, Berlin

[4] Morales-Morell A., 1997, "Intelligent Agents", http://citeseer.nj.nec.com/morales-morell97intelligent.html,

Tech paper, HCI course, unpublished paper

[5] Jennings, N. R., Wooldridge M., 1998. "Applications of Intelligent Agents", N.R Jennings and M. Wooldridge, eds., Agent Technology : Foundations, Applications, and Markets. Springer-Verlag, Berlin

[6] Skolicki Z., Kicinger R., 2002, "Intelligent Agent for Designing Steel Skeleton Structures of Tall Buildings", Proceedings, IT in CE Workshop, Washington DC

[7] Leitao, P., and Restivo, F., 2000, "A Framework for Distributed Manufacturing Applications." Proceedings, Advanced Summer Institute International Conference, ASI' 2000, Bordeaux, pp. 75-80.

[8] Wooldridge, M. J., and Jennings, N. R., 1995, "Intelligent Agents: Theory and Practice." The Knowledge Engineering Review, 10(2).

[9] Russell, S. J., and Norvig, P., 1995, “Artificial Intelligence: A Modern Approach”, Prentice Hall, New Jersey.

[10] Nwana, H. S., and Ndumu, D. T., 1999, "A Perspective on Software Agents Research", The Knowledge Engineering Review.

[11] Tecuci, G., 1998, "Building Intelligent Agents: An Apprenticeship Multistrategy Learning Theory, Methodology, Tool, and Case Studies”, Academic Press. 
[12] Stone P., Veloso M., 2000, "Multiagent Systems: A Survey from a Machine Learning Perspective", Autonomous Robots, vol. 8, no. 3

[13] Jennings N. et al., 1998, "A roadmap of agent research and development", Journal of Autonomous Agents and Multi-Agent Systems, 1, pp.275-306.

[14] Maher, M. L. and Fenves, S. J., 1984, "HI-RISE: A knowledge-based expert system for the preliminary structural design of high rise buildings", Report No. R-85-146, Department of Civil Engineering, Carnegie Mellon University.

[15] Pawlak, Z.W., 1991, "Rough Sets", Kluwer Academic Publishers, Dordrecht/Boston/London

[16] Arciszewski, T., Ziarko, W., 1988, "Adaptive Expert System for Preliminary Design of Wind Bracings in Skeleton Structures," chapter, in the book Second Century of Skyscrapers, van Nostrand Publishing Company, pp. 847-855

[17] Gero J. S., Brazier F. M. T., eds., 2002, "Agents in Design, Preprints of the First International Workshop on Agents in Design", University of Sydney

[18] Aly, S., Krishnamurti, R., 2002, "Can doors and windows become design team players?”, Gero J. S., Brazier F. M. T., eds., Agents in Design, Preprints of the First International Workshop on Agents in Design, University of Sydney, pp. 3-22

[19] Maher, M. L., Gu, N., 2002, "Design agents in virtual worlds", Gero J. S., Brazier F. M. T., eds., Agents in Design, Preprints of the First International Workshop on Agents in Design, University of Sydney, pp. 23-38

[20] Shumin Wu, Hamada Ghenniwa and Weiming Shen, 2002, "User model of a personal assistant in collaborative design environments", Gero J. S., Brazier F. M. T., eds., Agents in Design, Preprints of the First International Workshop on Agents in Design, University of Sydney, pp. 39-54

[21] Reffat, R. M., 2002, "Intelligent agents for concept invention of design forms", Gero J. S., Brazier F. M. T., eds., Agents in Design, Preprints of the First International Workshop on Agents in Design, University of Sydney, pp. 55-68

[22] Brazier, F. M. T., Wijngaards, N. J. E., 2002, "Role of trust in automated distributed design", Gero J. S., Brazier F. M. T., eds., Agents in Design, Preprints of the First International Workshop on Agents in Design, University of Sydney, pp. 7184

[23] Kannengiesser, U., Gero, J. S., 2002, "Situated agent communication" , Gero J. S., Brazier F. M. T., eds., Agents in Design, Preprints of the First International Workshop on Agents in Design, University of Sydney, pp. 85-94

[24] Klein, M., Sayama, H., Faratin, P., Bar-Yam, Y., 2002, “A complex systems perspective on how agents can support collaborative design", Gero J. S., Brazier F. M. T., eds., Agents in Design, Preprints of the First International Workshop on Agents in Design, University of Sydney, pp. 95-112

[25] Smith, G. J., Gero, J. S., 2002, "Interaction and experience" , Gero J. S., Brazier F. M. T., eds., Agents in Design, Preprints of the First International Workshop on Agents in Design, University of Sydney, pp. 115-132

[26] Saunders, R., Gero, J. S., 2002, "Curious agents and situated design evaluations", Gero J. S., Brazier F. M. T., eds., Agents in Design, Preprints of the First International Workshop on Agents in Design, University of Sydney, pp. 133-150

[27] Perrone, A., 2002, "Agent-based design modelling using the Visual Swarm Builder", Gero J. S., Brazier F. M. T., eds., Agents in Design, Preprints of the First International Workshop on Agents in Design, University of Sydney, pp. 153-164

[28] Sosa, R., Gero, J. S., 2002, "Cellular automata models of creative situations", Gero J. S., Brazier F. M. T., eds., Agents in Design, Preprints of the First International Workshop on Agents in Design, University of Sydney, pp. 165-180

[29] Moss, J., Kotovsky, K., Cagan, J., 2002, "Learning from design experience in an agent-based design system", Gero J. S., Brazier F. M. T., eds., Agents in Design, Preprints of the First International Workshop on Agents in Design, University of Sydney, pp. 181-200

[30] Hammond, T., Gajos, K., Davis R., Shrobe, H., 2002, “An agent-based system for capturing and indexing software design meetings", Gero J. S., Brazier F. M. T., eds., Agents in Design, Preprints of the First International Workshop on Agents in Design, University of Sydney, pp. 203-218

[31] Fujii, H., 2002, "Analysis of a retrospective discourse explaining an actual process of designing a house", Gero J. S., Brazier F. M. T., eds., Agents in Design, Preprints of the First International Workshop on Agents in Design, University of Sydney, pp. 219-231

[32] Liu, H., Tang, M., 2002, "A knowledge based collaborative design environment", Gero J. S., Brazier F. M. T., eds., Agents in Design, Preprints of the First International Workshop on Agents in Design, University of Sydney, pp. 232246

[33] Chen, Z. F., Brown, D. C., 2002, "Explorations of a two layered A-design system", Gero J. S., Brazier F. M. T., eds., Agents in Design, Preprints of the First International Workshop on Agents in Design, University of Sydney, pp. 249-256

[34] Liew, P. S., Gero, J. S., 2002, “An implementation model of constructive memory for a design agent", Gero J. S., Brazier F. M. T., eds., Agents in Design, Preprints of the First International Workshop on Agents in Design, University of Sydney, pp. 257-276

[35] Clarke, D.W., 2000, "Strategically Evolving the Future: Directed Evolution and Technological Systems Development," Arciszewski, T., guest editor, Special Issue of the International 
Journal Technological Forecasting and Social Change on "Innovation: the Key to Progress in Technological and Society, ", pp. 133-153

[36] Clarke, D.W., 2002, "TRIZ: Through the Eyes of an American TRIZ Specialist", Applied Innovation Alliance, LLC.

[37] Altshuller, G., 1984, "Creativity as an Exact Science: the Theory of he Inventive Problem Solving", Gordon and Breach Science Publishers, England.
[38] Terminko, J., Zusman, A., Zlotin, B., 1998, "Systematic Innovation: An Introduction to TRIZ”, St. Lucie Press.

[39] Arciszewski, T., Uduma, K., 1988, "Shaping of Spherical Joints in Space Structures." International Journal of Space Structures, Vol. 3, 3, pp. 171-183. 